\title{
Generalised soliton solutions of the Weyl class
}

\author{
$\mathrm{J}$ Carot $†$ and $\mathrm{E}$ Verdaguer $\ddagger$ \\ † Department of Mathematical Sciences, University of Aberdeen, The Edward Wright \\ Building, Dunbar Street, Aberdeen AB9 2TY, UK \\ $\ddagger$ Departament de Física Teòrica, Universitat Autònoma de Barcelona, E-08193 Bellaterra, \\ Barcelona, Spain
}

Received 25 March 1988, in final form 12 August 1988

\begin{abstract}
The whole family of generalised soliton solutions of the Weyl class for Einstein's equations in vacuum is considered. A classification scheme for the distinct types of metrics belonging to this family is given and emphasis is placed on the asymptotically flat solutions. The physical interpretation of the occurring parameters is also briefly considered.
\end{abstract}

\section{Introduction}

In the past years, a number of solution generation techniques have been developed to solve the vacuum Einstein field equations for spacetimes admitting two commuting non-null Killing fields. Solutions with an arbitrary number of parameters can be obtained by application of such techniques to simpler 'seed' solutions. The relations amongst the different known solution generation techniques have been given in some remarkable papers by Cosgrove $(1980,1982)$.

The Belinskii-Zakharov inverse scattering transformation (Belinskii and Zakharov 1978,1980 ), also called the 'soliton transformation', is one of those techniques which has proved particularly useful for the explicit evaluation of new solutions from old ones. It has been shown (Cosgrove 1980) that it is equivalent to Bäcklund transformations (Harrison 1978, Neugebauer 1979). The soliton transformations are characterised by the so-called 'pole trajectories'; that is, real or complex functions whose number determines the number of parameters of the new solution. There are two main contexts where this technique applies, namely the cosmological and the stationary and axisymmetric contexts.

It should be noticed that the term 'soliton' as used in this paper is in fact a loose expression which bears no clear relation to solitons in fluid dynamics (Korteweg-de Vries equation) or in quantum mechanics (sine-Gordon equation). In such cases soliton solutions are solutions of non-linear one-dimensional time-dependent equations, they show some localisability and shape persistence and can be found by the so-called 'inverse scattering transform'. The use of the term 'soliton solutions' associated with the Belinskii-Zakharov (BZ) technique stems from two main reasons. Firstly, there exists a certain formal relation between this technique and the inverse scattering transformation, although the $\mathrm{BZ}$ technique is not a true inverse scattering transformation since, for instance, the poles are not fixed numbers but functions. Secondly, it arises

§ On leave from: Departament de Física, Universitat Illes Balears, E-07071 Palma de Mallorca, Spain. 
from certain localisability and shape persistence properties possessed by some of the solutions obcained with the $\mathrm{BZ}$ technique in time-dependent spacetimes (admitting two spacelike Killing vectors). By extension, the term 'soliton solution' is also used in the literature for the stationary case (spacetimes admitting one spacelike and one timelike commuting Killing field). Thus the Schwarzschild metric is a two-soliton solution. After all, there is only a simple change of variables to go from one case (cosmological context; two spacelike Killing vectors) to the other (stationary and axisymmetric context; one spacelike and one timelike Killing vector).

In the cosmological case (both Killing vectors spacelike) Kitchingham $(1984,1986)$ has shown that a great number of known vacuum and stiff matter solutions can be systematically obtained by using the soliton transformation technique, either by itself or combined with some other transformations, from the anisotropic Kasner metric as seed solution. In the axisymmetric context, when one of the Killing vectors is timelike, some well known solutions such as the Kerr solution or the Kerr-NuT family of solutions are straightforwardly obtained from the Minkowski seed by using two real pole trajectories. When the Killing vectors are hypersurface orthogonal, the metric expressed in appropriate coordinates (e.g. Weyl coordinates) becomes diagonal; this implies that one of the Einstein equations, which is relevant for the soliton transformation, becomes linear.

It is worth noticing that, although the importance of the $\mathrm{BZ}$ technique (or others related such as the Bäcklund transformations) is that it allows solutions to be found in a systematic way to a non-linear system of equations which appears when nondiagonal metrics are considered, diagonal 'soliton' solutions can be obtained from the non-diagonal ones by taking some of the parameters in the $\mathrm{BZ}$ transformation to be null; the relevant equation of the resulting system is then linear and its solutions are then the 'soliton' solutions of the linear equation under consideration (whose general solution is known a priori, since it is linear). One can then view these diagonal soliton solutions as a limit of the non-diagonal soliton solutions, and expect them to share some of the properties of the latter.

The soliton solutions to this equation can be easily generalised thanks to the linearity of such equations. The generalisation is twofold. Firstly, the integer parameter which gives the degeneracy of the pole trajectories is taken to be a real number. Secondly, the real parameters which define the pole trajectories are extended into the complex plane yielding then a complex function whose real and imaginary parts, when considered separately, produce two independent real solutions (Céspedes and Verdaguer 1987). The new solutions so obtained are called 'generalised soliton solutions'.

In the cosmological context some well known solutions such as those of Wainwright et al (1979), the spatially inhomogeneous generalisations of the homogeneous Ellis and MacCallum (1969) cosmologies as well as the Carmeli and Charach (1980) pulse wave solutions, can be seen as generalised soliton solutions associated with the number of poles (Kitchingham 1984, Verdaguer 1985). Also new physically interesting solutions have been derived from the real and imaginary parts of complex poles in the cosmological context (Céspedes and Verdaguer 1987, Feinstein and Charach 1986, 1987) as well as in the cylindrically symmetric context (Garriga and Verdaguer 1987).

The present paper constitutes an attempt of classification of all the generalised soliton solutions in the axisymmetric context (i.e. Weyl class). We have tried to follow a systematic approach to all the possible cases occurring; to this end, we have given the Ernst potentials of all the solutions considered, thus making the identification of known solutions much easier, since the Ernst potential has been traditionally used to 
characterise stationary and axisymmetric solutions. Furthermore, the Ennst potential may be regarded as a complexified non-linear generalisation of the Newtonian potential (Kinnersley and Kelly 1974); thus helping the understanding of the physical meaning of the solutions.

In the real pole trajectories case, the generalised soliton solutions associated with a number of such poles give rise to well known solutions such as the Voorhees-Zipoy family (Voorhees 1970) and their superpositions (Cosgrove 1980, Tomimatsu and Sato 1981), which include superpositions of Schwarzschild black holes (Alekseev and Belinskii 1981). The generalised soliton solutions arising from the real and imaginary parts of complex pole trajectories have not been considered previously in this context; here they are dealt with in a similar way to that followed in the cosmological (Céspedes and Verdaguer 1987, Feinstein and Charach 1986, 1987) and cylindrically symmetric cases (Garriga and Verdaguer 1987).

The Levi-Civita (1919) metric is used throughout the paper as the seed metric; this metric can be interpreted as describing the gravitational field created by a massive line source and includes Minkowski flat spacetime as a special case. This metric is singular along the symmetry axis (except in the Minkowski flat spacetime case).

The physically most interesting axisymmetric solutions are the asymptotically flat ones since they can represent the gravitational field due to a finite source. Usually, the soliton solutions share the singularities of the seed, therefore the most interesting solutions will be those obtained from the Minkowski seed. Furthermore, a soliton solution involving a single pole trajectory (one soliton) introduces a new singularity at $z \rightarrow \infty$, but this singularity can be removed by the 'destructive' superposition of another 'opposite' pole ('antisoliton'). The asymptotically flat solutions, such as the Schwarzschild solution, are therefore constructed from the Minkowskian seed with a pair of opposite pole trajectories. Note that the two opposite poles must have different 'origins' on the symmetry axis, otherwise the seed solution is recovered; this, for instance, allows an interpretation of the Schwarzschild solution (in terms of Weyl coordinates) as the field due to a finite rod, whose mass and length are related to the soliton origins.

The paper is organised as follows: in $\S 2$ we recall explicitly the soliton solutions in terms of their Ernst potentials and classify the different types of solutions, that is one-soliton solutions and two-soliton (soliton-antisoliton) solutions in both real and complex pole trajectory cases, and also briefly describe the generalisations of these solutions. In $\S 3$ we deal with the first generalised type or 'generalised one-soliton solutions', which constitute the generalisation of the one-soliton solution, and with superpositions of solutions of the same type. Although most of these solutions are not asymptotically flat, as usually happens in solitonic solutions with a single pole (Verdaguer 1982), it turns out that those arising from the imaginary part of the pole trajectories (in the case of complex poles) are asymptotically flat, provided that the seed is flat. An attempt at physical interpretation of the parameters of the transformation is given based on the asymptotic behaviour of the Ernst potentials of the solutions under consideration. The $f$ coefficients (see (1)) have been given explicitly in all the solutions, that is, the one integration has been carried out explicitly, as it is typical of the $\mathrm{BZ}$ technique. This can help in the study of the properties of the solutions. The generalised two-soliton solutions (soliton-antisoliton pairs) and their superposition are described in $\S 4$. For the Minkowski seed such solutions are asymptotically flat. Amongst them we find the Voorhees-Zipoy metrics which are related to the solutions arising from real pole trajectories or from the real part of the pole trajectories in the 
case of complex poles. As before, a physical interpretation for the parameters of the transformation is provided and also the $f$ coefficients of the metric are given explicitly. On the other hand, the solutions obtained from the imaginary part of complex poles are also asymptotically flat and show a very similar behaviour to those coming from the imaginary part but in the case of a single pole. Again, some consideration is given to the physical meaning of the parameters of the transformation.

\section{Axisymmetric soliton solutions}

In this section we recall those Weyl solutions (static axisymmetric vacuum solutions) of the Einstein equations which can be obtained as soliton solutions. We also put forward a classification scheme for these solutions which will be developed throughout the paper.

Using Weyl coordinates, a general static axisymmetric vacuum solution of Einstein's equations can be written as

$\mathrm{d} s^{2}=f(\rho, z)\left(\mathrm{d} \rho^{2}+\mathrm{d} z^{2}\right)+\exp (-2 U(\rho, z)) \rho^{2} \mathrm{~d} \psi^{2}-\exp (2 U(\rho, z)) \mathrm{d} t^{2}$

and the corresponding field equations are then (Kramer et al 1980):

$$
\begin{aligned}
& U_{, z z}+(1 / \rho) U_{, \rho}+U_{, \rho \rho}=0 \\
& K_{, \rho}=\rho\left(U_{, \rho}^{2}-U_{, z}^{2}\right) \\
& K_{, z}=2 \rho U_{, \rho} U_{, z}
\end{aligned}
$$

where $K(\rho, z)$ is defined to be:

$$
K \equiv \frac{1}{2} \ln f+U
$$

The Ernst potential for such solutions is real and it is given by:

$$
\varepsilon=\mathrm{e}^{2 U} \text {. }
$$

Using the Levi-Civita solution as the seed (Levi-Civita 1919), the solitonic solutions of equation (1) can be written as (Belinskii and Zakharov 1979, Carr and Verdaguer 1983):

$$
\begin{aligned}
& U=\frac{1}{2}(d+1) \ln \rho+\frac{1}{2} \sum_{i=1}^{n} \ln \left( \pm \mu_{i}^{( \pm)} / \rho\right) \equiv U_{0}+U_{\mathrm{s}} \\
& f=A \rho^{\left(d^{2}-1\right) / 2} \rho^{n(4-n) / 2}\left[\prod_{k=1}^{n}\left(\mu_{k} / \rho\right)\right]^{2 d+n} \prod_{\substack{k, l=1 \\
k>l}}^{n}\left(\mu_{k}-\mu_{l}\right)^{2} \prod_{k=1}^{n}\left(\mu_{k}^{2}+\rho^{2}\right)
\end{aligned}
$$

where $d$ is the Levi-Civita parameter, $A$ is an integration constant and the functions $\mu_{i}^{ \pm}(\rho, z)$ are the so-called 'pole trajectories' defined by:

$$
\mu_{i}^{ \pm}=u_{i}-z \pm\left[\left(u_{i}-z\right)^{2}+\rho^{2}\right]^{1 / 2}
$$

$u_{i}$ being arbitrary complex parameters (note $\mu_{i}^{+} / \rho=-\rho / \mu_{i}^{-}$).

Since equation (2) is linear the solutions (5) are easily generalised by regarding $U$ as the superposition of different pole trajectories, the new solutions so obtained are called 'generalised soliton solutions'. This will be done in the next section. In order 
to see the general properties of these solutions, we shall restrict ourselves to the study of two different cases which summarise all the possibilities:

(a) one-pole solutions (one-soliton solutions) and their superposition:

(a1) real pole trajectories $\left(u_{i} \in \mathbb{R}\right)$

(a2) complex pole trajectories $\left(u_{i} \in \mathbb{C}\right)$;

(b) two-pole solutions (soliton-antisoliton solutions) and their superpositions:

(b1) real pole trajectories $\left(u_{i} \in \mathbb{R}\right)$

(b2) complex pole trajectories $\left(u_{i} \in \mathbb{C}\right)$.

Note that the cases $u_{i}$ complex, either in (a) or (b), would lead to complex solutions to Einstein's field equations which have no physical meaning; however, we shall use them to construct true physical solutions.

\section{Generalised one-soliton solutions}

In this section we will study and classify the generalised one soliton solutions and their superposition. We shall assume throughout the Levi-Civita metric as the seed metric.

\subsection{Real pole trajectories $\left(u_{i} \in \mathbb{R}\right)$}

We now take the parameters $u_{i}$ in (6) to be real. Since $U$ satisfies the linear equation (2) we can generalise the soliton solutions given in $(5 a)$ as:

$$
U_{\mathrm{s}}=\sum_{i=1}^{s} \frac{1}{2} h_{i} \ln \pm\left(\mu_{i}^{( \pm)} / \rho\right)=\sum_{i=1}^{s} \frac{1}{2} h_{i} \sinh ^{-1}\left[\left(u_{i}-z\right) / \rho\right]
$$

where the real parameters $h_{i}$ play the role of the degeneracy of the $i$ th pole when they are integers.

We can now integrate (3) to give explicitly the coefficient $f(\rho, z)$ appearing in (1). This is easily found from $(5 b)$ by taking the limit of $h_{i}$ 'degenerate' poles and choosing a suitable constant of integration $A$ :

$$
\begin{aligned}
f=A \rho^{\left(d^{2}-1-g^{2}\right) / 2} & \prod_{i=1}^{s}\left(\frac{\mu_{i}}{\rho}\right)^{h_{i}\left(2 h_{i}+d+g\right)}\left[1-\left(\frac{\mu_{i}}{\rho}\right)^{2}\right]^{-h_{i}^{2}} \\
& \times \prod_{\substack{i, j=1 \\
i>j}}^{s}\left(\left(\mu_{i}^{2}+\mu_{j}^{2}\right)+\frac{8 z_{i} z_{j} \mu_{i}^{2} \mu_{j}^{2}}{\left(\mu_{i}^{2}+\rho^{2}\right)\left(\mu_{j}^{2}+\rho^{2}\right)}\right)^{h_{i} h_{j}}
\end{aligned}
$$

where $z_{i} \equiv u_{i}-z$ and $g \equiv \sum_{i=1}^{s} h_{i}$.

The Ernst potential is then given by:

$$
\varepsilon=\rho^{d+1} \prod_{i=1}^{s}\left( \pm \mu_{i}^{ \pm} / \rho\right)^{h_{i}} .
$$

In order to physically understand these solutions we can consider just the one pole case, namely $s=1$; this solution is singular along the symmetry axis $\rho=0$ unless $d=-1$ (Minkowski seed) and at $z \rightarrow \infty$ (Verdaguer 1982); however, the singularity at $z \rightarrow \infty$ is not present in the particular case $h_{1}^{2}=d^{2}+3$, thus for $d=-1$ and $h_{1}^{2}=4$ the one-soliton metric is asymptotically flat. We note that this restriction shows up in the cosmological case, leading there to the Ellis and MacCallum (1969) spatially homogeneous solutions (Kitchingham 1984, Verdaguer 1985). 


\subsection{Complex pole trajectories $\left(u_{i} \in \mathbb{C}\right)$}

Let us now consider complex poles, i.e.

$$
u_{i}=z_{i}^{0}-\mathrm{i} w_{i}
$$

with $z_{i}^{0}$ and $w_{i}$ real arbitrary parameters and let us write the pole trajectories $\mu_{i}(\rho, z)$ as:

$$
\mu_{i}=\sqrt{\sigma_{i}} \rho \exp \left(\mathrm{i} \gamma_{i}\right)
$$

as they are solutions of the second-order equation

$$
\mu_{i}^{2}-2\left(u_{i}-z\right) \mu_{i}-\rho^{2}=0
$$

by plugging (11) into (12) and considering its complex conjugate, after some calculations we can come to

$$
\sin \gamma_{i}=-2 w_{i} \sqrt{\sigma_{i}} / \rho\left(\sigma_{i}+1\right) \quad \cos \gamma_{i}=2 z_{i} \sqrt{\sigma_{i}} / \rho\left(\sigma_{i}-1\right)
$$

and taking into account $\sin ^{2} \gamma_{i}+\cos ^{2} \gamma_{i}=1$ we get

$$
\sigma_{i}^{4}-\frac{4\left(w_{i}^{2}+z_{i}^{2}\right)}{\rho^{2}} \sigma_{i}^{3}+\left(\frac{8\left(w_{i}^{2}-z_{i}^{2}\right)}{\rho^{2}}-2\right) \sigma_{i}^{2}-\frac{4\left(w_{i}^{2}+z_{i}^{2}\right)}{\rho^{2}} \sigma_{i}+1=0
$$

which possesses two real solutions and two complex ones. The real solutions are

$$
\begin{aligned}
& \sigma_{i}^{ \pm}=L_{i} \pm\left(L_{i}^{2}-1\right)^{1 / 2} \quad\left(\sigma_{i}^{-}=\left(\sigma_{i}^{+}\right)^{-1}\right) \\
& L_{i} \equiv \frac{z_{i}^{2}+w_{i}^{2}}{\rho^{2}}+\left(1+2 \frac{z_{i}^{2}-w_{i}^{2}}{\rho^{2}}+\frac{\left(z_{i}^{2}+w_{i}^{2}\right)^{2}}{\rho^{4}}\right)^{1 / 2} .
\end{aligned}
$$

Note that in the real limit $\left(w_{i} \rightarrow 0\right)$ we recover the solutions in the case of real pole trajectories:

$$
\begin{aligned}
& L_{i}\left(w_{i} \rightarrow 0\right)=1+2\left(z_{i} / \rho\right)^{2} \\
& \sigma_{i}\left(w_{i} \rightarrow 0\right)=\left\{\left(z_{i} / \rho\right) \pm\left[\left(z_{i} / \rho\right)^{2}+1\right]^{1 / 2}\right\}^{2}=\left(\mu_{i}^{ \pm} / \rho\right)^{2} .
\end{aligned}
$$

These functions have the following limits:

$$
\begin{array}{ll}
\rho^{2} \gg z_{i}^{2} & \sigma_{i}^{-} \simeq 1-2\left|z_{i}\right| / \rho \\
z_{i}^{2} \gg \rho^{2} & \sigma_{i}^{-} \simeq \rho^{2} / 4 z_{i}^{2} \\
\rho^{2} \rightarrow 0 & \sigma_{i}^{-} \approx \frac{\rho^{2}}{2 w_{i}^{2}+z_{i}^{2}+\left(w_{i}^{4}+z_{i}^{4}\right)^{1 / 2}} .
\end{array}
$$

From (11) we have:

$$
\ln \mu_{i} / \rho=\ln \sqrt{\sigma_{i}}+\mathrm{i} \gamma_{i} .
$$

Recalling now $(5 a)$ and that $U$ satisfies a linear equation, we can regard (18) as furnishing two independent real solutions, one corresponding to the real part and the other one to the imaginary part of (18); these are the generalised soliton solutions with complex poles:

$$
\begin{array}{ll}
\text { class I } & U_{\mathrm{s}}^{(\mathrm{I})}=\sum_{i=1}^{s} \frac{1}{2} h_{i} \ln \sqrt{\sigma_{i}} \\
\text { class II } & U_{s}^{(1 \mathrm{I})}=\sum_{i=1}^{s} \frac{1}{2} h_{i} \gamma_{i}=\sum_{i=1}^{s} \frac{1}{2} h_{i} \cos ^{-1}\left(\frac{2 z_{i} \sqrt{\sigma_{i}}}{\rho\left(\sigma_{i}-1\right)}\right)
\end{array}
$$


where the arbitrary real parameters $h_{i}$ have again been introduced. The corresponding Ernst potentials are:

$$
\begin{array}{ll}
\text { class I } & \varepsilon^{(\mathrm{I})}=\rho^{d+1} \prod_{i=1}^{s} \sigma_{i}^{h_{i} / 2} \\
\text { class II } & \varepsilon^{(\mathrm{II})}=\rho^{d+1} \exp \left[\sum_{i=1}^{s} h_{i} \cos ^{-1}\left(\frac{2 z_{i} \sqrt{\sigma_{i}}}{\rho\left(\sigma_{i}-1\right)}\right)\right] .
\end{array}
$$

Let us now consider both classes of solutions separately.

Class I: these solutions clearly exhibit a behaviour similar to that of the generalised real pole solutions since, as has been pointed out, in the real limit $\left(w_{i} \rightarrow 0\right)$ both families of solutions coincide. They are singular along the symmetry axis $\rho=0$ and not asymptotically flat, excepting the special case of a single pole with $h_{1}^{2}=d^{2}+3$. The corresponding function $f(\rho, z)$ can be found either from $(5 b)$ or, more directly, by performing the appropriate changes from Garriga and Verdaguer (1987)

$$
\begin{aligned}
f^{(1)}=A \rho^{\left(d^{2}-1-g^{2}\right) / 2} & \prod_{i=1}^{s} \sigma_{i}^{h_{i}\left(2 h_{i}+d+g\right) / 2}\left(1-\sigma_{i}\right)^{-h_{i}^{2} / 2} H_{i}^{-h_{i}^{2} / 4} \\
& \times \prod_{\substack{i, j=1 \\
i>j}}^{s}\left[\left(\left(\sigma_{i}+\sigma_{j}\right) \rho^{2}+\frac{8 z_{i} z_{j} \sigma_{i} \sigma_{j}}{\left(1+\sigma_{i}\right)\left(1+\sigma_{j}\right)}\right)^{2}-\frac{64 w_{i}^{2} w_{j}^{2} \sigma_{i}^{2} \sigma_{j}^{2}}{\left(1-\sigma_{i}\right)^{2}\left(1-\sigma_{j}\right)^{2}}\right]^{h_{i} h_{j} / 2}
\end{aligned}
$$

where

$$
H_{i} \equiv\left(1-\sigma_{i}\right)^{2}+16 w_{i}^{2} \sigma_{i}^{2} \rho^{-2}\left(1-\sigma_{i}\right)^{-2}
$$

For a single pole and when $w_{i} \neq 0$, it is possible to define a set of new coordinates so that $\sigma_{i}$ takes a much simpler expression than the one in terms of Weyl coordinates given in (15). We define the new coordinates as:

$$
\begin{aligned}
& \rho \equiv w_{i} \cosh \left(2 a_{i} \hat{R}_{i}\right) \sin \hat{\theta}_{i} \\
& z_{i} \equiv w_{i} \sinh \left(2 a_{i} \hat{R}_{i}\right) \cos \hat{\theta}_{i}
\end{aligned}
$$

where $a_{i}$ are arbitrary real parameters. In these new coordinates $\sigma_{i}$ reads now:

$$
\sigma_{i}^{+}=\frac{1+\cos \hat{\theta}_{i}}{1-\cos \hat{\theta}_{i}} \quad \sigma_{i}^{-}=\left(\sigma_{i}^{+}\right)^{-1} .
$$

Note also that in this case the line element $\mathrm{d} \rho^{2}+\mathrm{d} z^{2}$ transforms itself into:

$$
\mathrm{d} \rho^{2}+\mathrm{d} z^{2}=w_{i}^{2}\left(\sinh ^{2}\left(2 a_{i} \hat{R}_{i}\right)+\cos ^{2} \hat{\theta}_{i}\right)\left\{4 a_{i}^{2} \mathrm{~d} \hat{R}_{i}^{2}+\mathrm{d} \hat{\theta}_{i}^{2}\right\}
$$

still keeping its diagonal form.

To see the geometrical meaning of these coordinates, we can consider their behaviour at large distances; assuming (at large distances) a relationship between Weyl and spherical coordinates of the type:

$$
\begin{aligned}
& \rho=r \sin \theta \\
& z_{i}^{0}-z \equiv z_{i}=r \cos \theta
\end{aligned}
$$

the new coordinates behave then as

$$
\tan \hat{\theta}_{i} \sim \rho / z_{i} \quad \exp \left(4 a_{i} \hat{R}_{i}\right) \sim\left(1 / w_{i}\right)\left(\rho^{2}+z_{i}^{2}\right)
$$

i.e. $\hat{\theta}_{i}$ behaves as a spherical angular coordinate and $\hat{R}_{i} \sim \ln r$. 
Class II: these are the solutions arising from the imaginary part of (18). Their behaviour is quite different from that of those previously considered (class I). In the real limit $w_{i} \rightarrow 0$ we get $\gamma_{i}^{ \pm} \rightarrow 0$ which does not produce any new solution. The function $f(\rho, z)$ corresponding to this case can be found by the same procedure as in the former case (Garriga and Verdaguer 1987):

$$
\begin{aligned}
f^{(1)}=A \rho^{\left(d^{2}+2 g-1\right) / 2} & \prod_{\substack{i, j=1 \\
i>j}}^{s}\left(A_{i j}^{-} / A_{i j}^{+}\right)^{h_{i} h_{j} / 2} \exp \left(d \sum_{i=1}^{s} h_{i} \gamma_{i}\right) \prod_{i=1}^{s} \sigma_{i}^{h_{i}\left(h_{i}+1\right) / 2} \\
& \times\left\{\left(2 z_{i}^{2}-w_{i}^{2}-\rho^{2}\right)^{2}+4 w_{i}^{2} z_{i}^{2}\right\}^{h_{i}\left(h_{i}+1\right) / 4}\left(\sigma_{i}+1\right)^{-h_{i}^{2} / 2} H_{i}^{-h_{i}\left(h_{i}+2\right) / 4} \rho^{-h_{i}^{2}}
\end{aligned}
$$

with $A_{i j}^{ \pm}$given by:

$\boldsymbol{A}_{i j}^{ \pm}=\left(\sigma_{i}+\sigma_{j}\right)\left(1-\sigma_{i}^{2}\right)\left(1-\sigma_{j}\right)^{2} \rho^{2}-8 \sigma_{i} \sigma_{j}\left\{z_{i} z_{j}\left(\sigma_{i}+1\right)\left(\sigma_{j}+1\right) \pm w_{i} w_{j}\left(1-\sigma_{i}\right)\left(1-\sigma_{j}\right)\right\}$.

In the case of a single pole, we can use again the coordinates introduced in (25) in order to simplify the expression of $\gamma_{i}$, we then have

$$
\gamma_{i}^{ \pm}=\cos ^{-1}\left\{ \pm \tanh \left(2 a_{i} \hat{R}_{i}\right)\right\} \quad \gamma_{i}^{-}=\pi \pm \gamma_{i}^{+} .
$$

To physically understand these solutions (Kinnersley and Kelley 1974), we again take the single-pole case and asymptotically expand its associated Ernst potential $\left(\varepsilon=\exp \left(2 \gamma_{i}^{+}\right)\right)$in a power series of $r^{-1}$. Then, at large distances we have

$$
\varepsilon=-\frac{\sqrt{2}}{2 \cos \theta}\left\{1-\left(1+4 w_{i}^{2} \cos ^{2} \theta\right)^{1 / 2}\right\} \frac{1}{r}+\mathrm{O}\left(\frac{1}{r^{2}}\right)
$$

where we have assumed the same relationship between both Weyl and spherical coordinates as in (27).

The last expression shows that these solutions are asymptotically flat, provided the seed is (this can also be seen directly from the expression for $\gamma_{i}$ ). However, no classical interpretation is possible here, since even at the first order in $r^{-1}$ an angular dependence appears. For this reason, one cannot interpret the real parameters $w_{k}$ as related to the mass of the object creating the gravitational field, in spite of their occurrence at first order.

\section{Generalised two-soliton solutions}

In this section we shall consider the generalised soliton solutions obtained by the superposition of soliton-antisoliton pairs. As before, we shall deal separately with the real pole trajectory case and the complex pole trajectory one. It will be shown that the divergences at infinity appearing in some of the previous cases are no longer present now as a consequence of the combination soliton-antisoliton. The solutions so obtained are therefore asymptotically flat and can be expanded, at large distances, in a power series of $r^{-1}$ which eventually will allow us to figure out the physical meaning of the different parameters appearing in these solutions.

\subsection{Real pole trajectories $\left(u_{i} \in \mathbb{R}\right)$}

We shall now consider pairs of (real) pole trajectories $\left(\mu_{i}^{+}, \mu_{i+1}^{-}\right)$(i.e. soliton-antisoliton) with the coefficients $h_{k}$ occurring in $(5 a, b)$ satisfying $h_{i+1}=-h_{i}$. For the 
superposition of $s$ such pairs of poles we have, from $(5 a)$ :

$$
U_{\mathrm{s}}=\frac{1}{2} \sum_{i=1}^{s} h_{i}\left\{\ln \left(-\mu_{i}^{-} / \rho\right)-\ln \left(-\mu_{\overline{i+1}}^{-} / \rho\right)\right\}
$$

where use has been made of the equality $\mu_{k}^{+} / \rho=-\left(\mu_{k}^{-} / \rho\right)^{-1}$.

The Ernst potential is then, assuming the Levi-Civita metric as the seed,

$$
\varepsilon=\rho^{d+1} \prod_{i=1}^{s}\left(\frac{\mu_{i}^{-}}{\mu_{i+1}^{-}}\right)^{h_{i}} .
$$

These solutions, characterised by their Ernst potentials (33), can be written in more familiar forms by changing Weyl coordinates to prolate spheroidal or to BoyerLindquist coordinates.

Using prolate spheroidal coordinates $x_{i}, y_{i}$, defined by

$$
\rho^{2}=a_{i}^{2}\left(x_{i}^{2}-1\right)\left(1-y_{i}^{2}\right) \quad z-z_{i}=a_{i} x_{i} y_{i}
$$

where $a_{i}$ is (Tomimatsu and Sato 1981)

$$
u_{i}=z_{i}+a_{i} \quad u_{i+1}=z_{i}-a_{i}
$$

the Ernst potential (33) can be written as:

$$
\varepsilon=-\rho^{d+1} \prod_{i=1}^{s}\left(\frac{x_{i}-1}{x_{i}+1}\right)^{h_{i}} .
$$

This family of solutions includes that of Voorhees-Zipoy (Voorhees 1970) $(s=1$, i.e. 2 poles, $d=-1$ and $h_{1}=\delta$ ) which in its turn includes the Schwarzschild solution as a special case $\left(\delta=h_{1}=1\right)$ (Kramer et al 1980). Note that while Voorhees-Zipoy metrics generally have an Abelian symmetry group $\mathrm{G}_{2}$, the Schwarzschild metric has a larger symmetry group $\mathrm{G}_{4}$. For $s>1, h_{i}=1$; we get a superposition of Schwarzschild black holes along the $z$ axis.

Using Boyer-Lindquist coordinates (Belinskii and Zakharov 1980),

$$
\rho=\left[r_{i}\left(r_{i}-2 m_{i}\right)\right]^{1 / 2} \sin \theta_{i} \quad z-\hat{z}_{i}=\left(r_{i}-m_{i}\right) \cos \theta_{i}
$$

with $m_{i}$ and $\hat{z}_{i}$ defined as

$$
m_{i} \equiv \frac{1}{2}\left(u_{i+1}-u_{i}\right) \quad \hat{z}_{i} \equiv \frac{1}{2}\left(u_{i+1}+u_{i}\right)
$$

the Ernst potential (33) takes now the form:

$$
\varepsilon=-\rho^{d+1} \prod_{i=1}^{s}\left(1-2 m_{i} / r_{i}\right)^{h_{i}}
$$

To see the physical meaning of these solutions and their asymptotic behaviour, we consider the case $s=1$ (a single pair of poles) and as in the previous cases expand the solitonic part of the Ernst potential (now $\mu_{i}^{-} / \mu_{i+1}^{-}=\rho^{-2} \mu_{i}^{-} \mu_{i+1}^{+}$) in a power series of $r^{-1}$ (the same relation between Weyl and spherical coordinates at large distances as in (27) will be assumed here); we then have:

$$
\rho^{-2} \mu_{i}^{-} \mu_{i+1}^{+}=1-4 m_{i}\left(1 / r_{i}\right)+\mathrm{O}\left(1 / r_{i}^{2}\right)
$$

which displays clearly the asymptotic flatness of the solution (provided the seed is flat) and also shows that, at first order, we recover the Schwarzschild solution for a mass $2 m_{i}$. 


\subsection{Complex poles $\left(\boldsymbol{u}_{i} \in \mathbb{C}\right)$}

As in the previous section, we now take complex pole parameters $u_{i}=z_{i}^{0}-\mathrm{i} w_{i}\left(z_{i}^{0}, w_{i} \in \mathbb{R}\right)$ and write the complex pole trajectories as $\mu_{i}=\sqrt{\sigma}_{i} \rho \exp \left(\mathrm{i} \gamma_{i}\right)$ with $\sigma_{i}^{ \pm}$and $\gamma_{i}^{ \pm}$given by (13) and (15) respectively. Recalling now (18), we shall deal separately with the solutions arising from the real and the imaginary parts of the complex pole trajectories, i.e. class I (19) and class II (20) solutions, taking now, in each case, the superposition of two solutions of the same class but different signs (soliton-antisoliton pair) and with the pole 'degeneracies' $h_{k}$ satisfying $h_{i+1}=-h_{i}$ as before. We then have, for $s$ pairs of poles:

$$
\begin{array}{ll}
\text { class I } & U_{\mathrm{s}}^{(\mathrm{I})}=\frac{1}{2} \sum_{i=1}^{s} h_{i}\left[\ln \left(\sigma_{i}^{-}\right)^{1 / 2}-\ln \left(\sigma_{i+1}^{-}\right)^{1 / 2}\right] \\
\text { class II } & U_{\mathrm{s}}^{(\mathrm{II})}=\frac{1}{2} \sum_{i=1}^{s} h_{i}\left(\gamma_{i}^{+} \pm \gamma_{i+1}^{+}\right) .
\end{array}
$$

Note that class II solutions admit two different possibilities of combination of the two $\gamma$ functions, both leading-as we shall see later on-to asymptotically flat solutions. One could think of a third possibility for the combination of the $\gamma$ functions, namely $\gamma_{i}^{-}-\gamma_{i+1}^{-}$, but taking (30) into account, it is easy to see that it coincides with (42).

We shall now consider each class separately, paying special attention to their asymptotic behaviour as this provides a physical interpretation of the parameters $w_{i}$.

We shall use either Weyl coordinates or those introduced in (25) which seem to be especially well adapted to this case. When considering asymptotic behaviour, we shall use Boyer-Lindquist coordinates (37); that is, for each pair of poles:

$$
\rho=\left[r_{i}\left(r_{i}-2 m_{i}\right)\right]^{1 / 2} \sin \theta_{i} \quad z-\hat{z}_{i}=\left(r_{i}-m_{i}\right) \cos \theta_{i}
$$

with $m_{i}$ and $\hat{z}_{i}$ now defined as:

$$
m_{i} \equiv \frac{1}{2}\left(z_{i}^{0}-z_{i+1}^{0}\right) \quad \hat{z}_{i} \equiv \frac{1}{2}\left(z_{i}^{0}+z_{i+1}^{0}\right) .
$$

At large distances, these coordinates become simply

$$
\rho=r_{i} \sin \theta_{i} \quad z-\hat{z}_{\mathrm{i}}=r_{i} \cos \theta_{i}
$$

and then

$$
z_{i}=m_{i}-r_{i} \cos \theta_{i} \quad z_{i+1}=-\left(m_{i}+r_{i} \cos \theta_{i}\right)
$$

Class I: taking now (41) into account and assuming the Levi-Civita metric as seed, the Ernst potential corresponding to the superposition of $s$ pairs of poles $\left(\sigma_{i}^{-}, \sigma_{i+1}^{+}\right)$ in the above described way, is

$$
\varepsilon=\rho^{d+1} \prod_{i=1}^{s}\left(\sigma_{i}^{-} \sigma_{i+1}^{+}\right)^{h_{i}} .
$$

This expression reduces to (33) when $w_{i} \rightarrow 0$ and includes therefore a superposition of Schwarzschild black holes when $d=-1$ and $h_{i}=1$. In order to see the physical meaning of the parameters $m_{i}$ and $w_{i}$ appearing in these solutions, let us restrict ourselves to the case of a single pair of poles (soliton-antisoliton) and expand $\sigma_{i}^{-} \sigma_{i+1}^{+}$at large distances in a power series of $r_{i}^{-1}$ according to its definition $(43 c)$. We then get

$$
\sigma_{i}^{-} \sigma_{i+1}^{+} \simeq 1-4 m_{i} r_{i}^{-1}+\left\{8 m_{i}^{2}+\left(w_{i+1}^{2}-w_{i}^{2}\right) \cos \theta\right\} r_{i}^{-2}+\ldots
$$


This expression shows the asymptotic flatness of the solution (for a flat seed). We can also see that, for first order in $r_{i}^{-1}$, it coincides with (40), reproducing the Schwarzschild potential for a mass $2 m_{i}$ as should be expected (note that there is no dependence on $w_{k}$ for first order). The second order contains the parameters $w_{k}$ in a way that suggests they are related to the dipolar moment; in particular, if we shift the origin of distances an amount $2 m_{i}$ (i.e. $r_{i}=r_{i}^{\prime}-2 m_{i}$ ) then the term $8 m_{i}^{2}$ appearing in the second order of the expansion in terms of $r_{i}^{-1}$ cancels out with another term appearing when the expansion is performed with respect to the new variable $r_{i}^{\prime-1}$, being then:

$$
\sigma_{i}^{-} \sigma_{i+1}^{+} \simeq 1-4 m_{i} r_{i}^{\prime-1}+\left(w_{i+1}^{2}-w_{i}^{2}\right) \cos \theta r_{i}^{\prime-2}+\ldots
$$

and therefore $w_{i+1}^{2}-w_{i}^{2}$ is exactly the dipolar moment. For further references about higher degree multipoles see, for instance, Cosgrove (1980) and Hoenselaers (1976).

In the coordinates $\hat{R}_{i}, \hat{R}_{i+1}, \hat{\theta}_{i}, \hat{\theta}_{i+1}$ introduced in (25) (note that these coordinates $\hat{R}_{k}, \hat{\theta}_{k}$ are adapted to a single pole while $r_{i}, \theta_{i}$ were adapted to a pair of poles), the product $\sigma_{i}^{-} \sigma_{i+1}^{+}$can be written as:

$$
\sigma_{i}^{-} \sigma_{i+1}^{+}=1+2 \frac{\cos \hat{\theta}_{i}-\cos \hat{\theta}_{i+1}}{\left(1+\cos \hat{\theta}_{i}\right)\left(1-\cos \hat{\theta}_{i+1}\right)}
$$

which again shows the asymptotic flatness of the solution (assuming a flat seed).

Class II: this class of solutions arises from the superposition of two $\gamma_{k}$ functions and, as was previously pointed out, there are two different possibilities of combination, both of them leading to asymptotically flat solutions (assuming a flat seed). From (42) and taking the Levi-Civita metric as the seed, the Ernst potential corresponding to the superposition of $s$ pairs of poles is

$$
\varepsilon=\rho^{d+1} \exp \left(\sum_{i=1}^{s} h_{i}\left(\gamma_{i}^{+} \pm \gamma_{i+1}^{+}\right)\right) .
$$

Again, note that when $w_{i} \rightarrow 0$ no new solutions are obtained, since in this case $\gamma_{k}^{ \pm} \rightarrow 0$, and therefore we reobtain the seed metric.

The asymptotic flatness of these solutions follows immediately from (47) together with the expression (30) for the $\gamma_{k}$ functions.

As in the previous cases, and in order to see in some detail the asymptotic behaviour of these solutions and discuss the physical interpretation of the parameters $w_{k}$, we shall take a single pair of poles $\gamma_{i}^{+} \pm \gamma_{i+1}^{+}$and expand the corresponding Ernst potential (Kinnersley and Kelley 1979) in a power series of $r_{i}^{-1}$ at large distances; assuming for $r_{i}$ and $\theta_{i}$ the expression given in (43), we have

$\varepsilon \simeq-\frac{\sqrt{2}}{2 \cos \theta_{i}}\left\{\left[1-\left(1+4 w_{i}^{2} \cos ^{2} \theta_{i}\right)^{1 / 2}\right] \pm\left[1-\left(1+4 w_{i+1}^{2} \cos ^{2} \theta_{i}\right)^{1 / 2}\right]\right\} r_{i}^{-1}+\ldots$

As we can straightforwardly see, these solutions do not reproduce the Schwarzschild potential at first order, they are therefore clearly different from those of class I. There are two possible different solutions, depending on the choice of sign we make in the former expression, and both are asymptotically flat. However, the same remarks as in the case of a single pole (31) apply here, in the sense that no classical interpretation is possible here because of the angular dependence at first order and, therefore, no clear interpretation of the parameters $w_{k}$ in terms of the mass of the object creating the gravitational field is possible either, despite the fact that they appear at first order. 
Note that, according to the preceding comments, these soliton-antisoliton class II solutions are very closely related to the one-soliton class II solutions, both verifying analogous properties and exhibiting similar behaviours. This is completely different from what happens with soliton-antisoliton and one-soliton class I solutions; the first being formed by destructive superposition of two of the latter and each displaying very different behaviour; in particular, the one-soliton class I solutions diverge at infinity (except in a very particular case), while the soliton-antisoliton ones are asymptotically flat (provided that the seed metric is fiat).

\section{Acknowledgments}

We are grateful to Dr D Kramer from FSU Jena and to Dr L L Mas from UIB for very fruitful discussions. Financial support from 'Conveni UAB-UIB' and from CAICYT research project PR84-1005 is also acknowledged. One of us (JC) wishes to thank Dr G S Hall for his hospitality during his stay at the University of Aberdeen where this paper was written.

\section{References}

Alekseev G A and Belinskii V A 1981 Sov. Phys.-JETP 51655

Belinskii V A and Zakharov V E 1978 Sov. Phys.-JETP 48985

1980 Sov. Phys.-JETP 501

Carmeli M and Charach Ch 1980 Phys. Lett. 75A 333

Carr B J and Verdaguer E 1983 Phys. Rev. D 282995

Céspedes J and Verdaguer E 1987 Class. Quantum Grav. 4 L7

Cosgrove C M 1980 J. Math. Phys. 212417

1982 J. Math. Phys. 23615

Ellis G F R and MacCallum M A H 1969 Commun. Math. Phys. 12108

Feinstein A and Charach Ch 1986 Class. Quantum Grav. 3 L5

1987 Preprint Ben Gurion University of the Negev, Israel

Garriga J and Verdaguer E 1987 Phys. Rev. D 362250

Harrison B K 1978 Phys. Rev. Lett. 41119

Hoenselaers C 1976 Prog. Theor. Phys. 56324

Kinnersley W and Kelly E F 1974 J. Math. Phys. 152121

Kitchingham D W 1984 Class. Quantum Grav. 1677

1986 Class. Quantum Grav. 3133

Kramer D, Stephani H, MacCallum M and Herlt E 1980 Exact Solutions of Einstein's Field Equations (Cambridge: Cambridge University Press)

Levi-Civita T 1919 Rend. Acc. Lincei 283

Neugebauer G 1979 J. Phys. A: Math. Gen. 12 L67

Tomimatsu A and Sato H 1981 Prog. Theor. Phys. 70215

Verdaguer E 1982 J. Phys. A: Math. Gen. 151261

Verdaguer E 1985 Observational and Theoretical Aspects of Relativistic Astrophysics and Cosmology ed J L Sanz and L J Goicoechea (Singapore: World Scientific) p 311

Voorhees B H 1970 Phys. Rev. D 22119

Wainwright J, Ince W C W and Marshman B 1979 Gen. Rel. Grav. 10259 